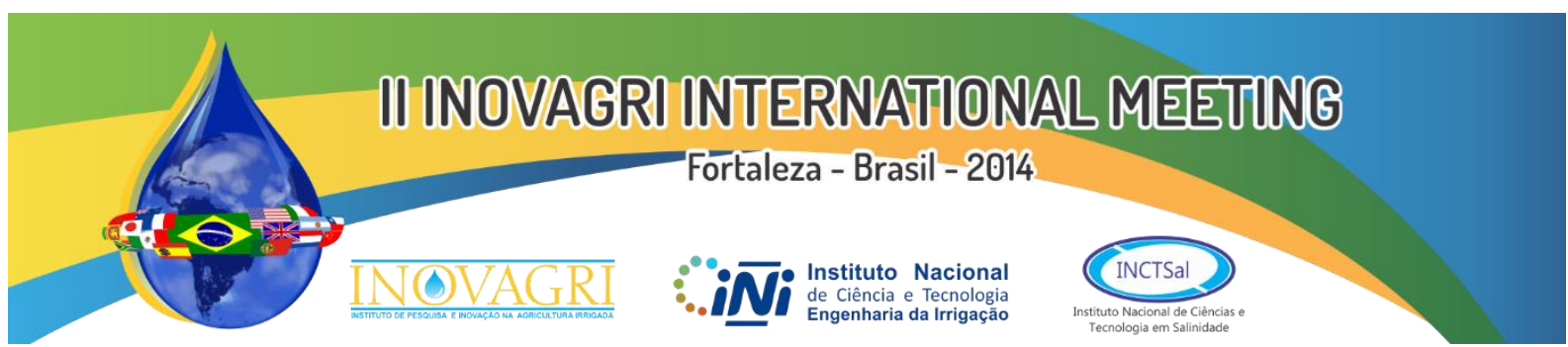

http://dx.doi.org/10.12702/ii.inovagri.2014-a712

\title{
UNIFORMIDADE DE DISTRIBUIÇÃO DE ÁGUA E IONS EM DIFERENTES PROFUNDIDADES DO SOLO COM BANANEIRA FERTIRRIGADA
}

\author{
F. P. SANTOS ${ }^{1}$, E. F. COELHO ${ }^{2}$, J.T. DE CARVALHO ${ }^{1}$
}

\begin{abstract}
RESUMO: A uniformidade de distribuição (CUD), de água e nutrientes via fertirrigação por microaspersão em bananeira tem sido avaliada em condições de solo nu, entretanto, é necessário avaliar com o solo sob cobertura morta, o que altera as propriedades físicas do solo nessa condição. Esse trabalho teve como objetivo avaliar a uniformidade de distribuição de água, da Condutividade elétrica aparente (CEa), dos íons potássio e nitrato no entorno do microaspersor após uma fertirrigação em um pomar de bananeira (BRS Princesa). O experimento seguiu o delineamento de blocos casualizados em esquemas de parcelas subdivididas, com os tratamentos T1: com cobertura e T2: sem cobertura na parcela, profundidade de 0,20 0,40 e 0,60 m na sub parcela. As avaliações foram feitas 1 e 24 horas após a fertirrigação. A avaliação foi feita com sondas de TDR instaladas a 0,20; 0,40 e $0,60 \mathrm{~m}$, próximo de coletores de água dispostos em uma malha de 0,50 x 0,50 m com o microaspersor de vazão $53 \mathrm{Lh}^{-1}{ }^{1}$ instalado entre quatro plantas. $\mathrm{O}$ tratamento com cobertura morta apresentou maior coeficiente de uniformidade de distribuição (CUD) em todas as profundidades comparado ao tratamento sem cobertura enquanto a condutividade elétrica teve comportamento inverso onde o CUD foi superior no tratamento sem cobertura.. Após a fertirrigação a umidade do solo com cobertura foi estatisticamente superior a do sem cobertura, entretanto esses valores se aproximaram 24 horas após.
\end{abstract}

PALAVRAS CHAVE: Coeficiente de uniformidade de distribuição, Fertirrigação, Microaspersão.

\section{WATER AND ION DISTRIBUTION UNIFORMITY AT DIFFERENT SOIL DEPTHS WITH FERTIRRIGATED BANANA}

ABSTRACT: Water and ion distribution uniformity (CUD), have been evaluated for bare soil. However, it is necessary to evaluate CUD for condition of using mulch. This work had as objective to evaluate water $\mathrm{CEa}$, potassium and nitrate distribution uniformity around a microsprinkler after a fertirrigation in a banana (BRS Princesa).area. The experiment followed a random block design, in split plot scheme with treatments T1 with soil cover and T2 without cover in the plot, depth of 0.20 ; 0.40 e $0.60 \mathrm{~m}$ in the split plot. Evaluations were done 1 and 24 hours after irrigation. The evaluation was done by using TDR probes inserted at $0.20 ; 0.40$ e $0.60 \mathrm{~m}$ depths close to water collectors in grid of $0.5 \mathrm{~m} \mathrm{x} 0.5 \mathrm{~m}$ with the microsprinkler of $53 \mathrm{~L} \mathrm{~h}-1$ flow rate located among four plants. Treatment with cover (mulch) showed larger CUD for all depths compared to the bare soil treatment while electric conductivity showed an inverse behavior where CUD was larger for bare soil. Soil water content in treatment with soil cover was statistically larger than water content in bare soil after fertirrigation, however these values got close in the next 24 hours.

Key Words: Distribution uniformity coefficient, fertirrigation, microsprinkler

\section{INTRODUÇÃO}

O uso da água na agricultura representa, a nível mundial, cerca de $70 \%$ de toda a água doce, enquanto a indústria utiliza $23 \%$ e o abastecimento humano, $7 \%$ (SANTOS 1998). Dessa forma se faz necessário a utilização dessa prática com maior eficiência em vista à escassez de água em nossos

\footnotetext{
${ }^{1}$ Estudante de Agronomia,Graduando, UFRB/Cruz das Almas - BA, Fone: (075)92313625, fpsagro@gmail.com 2 Eng $^{\circ}$ Agricola, Pesquisador.Dr.,Embrapa Mandioca e Fruticultura(CNPMF)/Cruz das Almas - BA
}

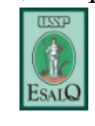


mananciais, evidenciando assim sua grande importância. A uniformidade de distribuição é uma informação importante para avaliar o desempenho dos sistemas de irrigação localizada, variabilidade da lâmina de água na superfície irrigada e na fertirrigação, a distribuição uniforme dos nutrientes à planta. Segundo FRIZZONE (1992) o coeficiente de uniformidade de distribuição da água e a eficiência de aplicação são os principais parâmetros utilizados na avaliação do sistema de irrigação por aspersão, pois expressam a qualidade da irrigação e são decisivos no planejamento e na operação desse sistema. Proposto por ASAE (1996), o procedimento para a avaliação da uniformidade da distribuição de água dos sistemas de irrigação mostram valores Excelente de CUD entre 94- 100\%, Bom 81-87\%, Normal 68-75\%, Ruim 56-62\% e Inaceitável > 50\%. Segundo LÓPEZ et al. (1992), na atualidade, em sistemas de irrigação localizada, é mais frequente o uso do coeficiente de uniformidade de distribuição, pois ele possibilita uma medida mais restrita, dando maior peso às plantas que recebem menos água. A produtividade tende a aumentar com a uniformidade de irrigação SOARES et al. (1993). Este trabalho teve como objetivo avaliar o CUD da distribuição de umidade e da condutividade elétrica aparente do solo na superfície do solo em diferentes profundidades em um pomar de bananeira (BRS princesa). Normalmente sistemas de irrigação localizados apresentam altos índices de eficiência de aplicação, pois são bem menores comparados a aspersão devido a aplicação ser pontual e segundo AZEVEDO (1986) quando bem projetada e manejada, a área máxima molhada não deve ser superior a 55\% da área sombreada pela planta, com área mínima molhada de $20 \%$ nas regiões úmidas e de 30\% nas regiões de clima semi-árido (Azevedo, 1986). BERNARDO (2008) afirma que a uniformidade da irrigação tem efeito no rendimento das culturas, sendo considerado um dos fatores mais importantes na operação de sistemas de irrigação. Segundo SILVA \& SILVA (2005) para que a irrigação seja eficiente, é imperativo que os sistemas apresentem alta uniformidade de aplicação da água. Uma vez instalado um projeto de irrigação, é necessário verificar se as condições previstas inicialmente se confirmam em campo. Para tanto, deve-se avaliar as condições de pressão, vazão e lâminas d'água aplicadas. Na realidade, a avaliação de sistemas de irrigação é um tema que os agricultores pouco têm dado importância. Mesmo tendo acesso à tecnologia, muitos não a utilizam de forma adequada, por falta de orientação e conhecimento. Uma das dificuldades encontrada na irrigação localizada reside na facilidade que as secções mais finas dos emissores têm em se obstruírem, podendo afetar a distribuição da água para as plantas e consequentemente a produção. O sistema de irrigação pode ser avaliado levando-se em conta aspectos técnicos (eficiência e uniformidade) e aspectos econômicos (SOUZA, 2001). JENSEN ET AL. (1967) afirmam que, conhecendo-se o perfil de distribuição dos aspersores, pode-se avaliar a adequação de uma irrigação, ou mesmo ajustar a sua duração, obtendo-se a lâmina desejada. Por razões econômicas, parte da área pode ser subirrigada, enquanto o restante da área recebe irrigação adequada. A porcentagem da área sub-irrigada depende do valor econômico e da sensibilidade das plantas à deficiência de água ou excesso. A ABNT (1985) recomenda, no seu projeto de norma 12:02.08-005, a análise da distribuição de água por meio da uniformidade na porção da área irrigada, que recebe menos água. O coeficiente de uniformidade de distribuição (CUD) é definido como a medida da distribuição da água que relaciona a quarta parte da área irrigada total, que recebe menos água, com a lâmina média aplicada. Caso toda a área receba no mínimo a lâmina real necessária, um baixo valor de CUD indica excessiva perda por percolação. REZENDE ET AL. (1992) analisaram uniformidade de distribuição acima e abaixo da superfície do solo irrigado por pivô-central, utilizando os coeficientes CUC e CUD e concluíram que, em todas as profundidades, os coeficientes de uniformidade foram superiores aos obtidos acima da superfície do solo. Os valores dos coeficientes de uniformidade aumentaram com o tempo em todas as profundidades. Os altos valores alcançados pelos coeficientes de uniformidade abaixo da superfície do solo mostraram que a uniformidade abaixo da superfície é sempre maior que a uniformidade acima. PAIVA (1980) trabalhou com um sistema de irrigação por aspersão convencional, para analisar o efeito da uniformidade solo, após a redistribuição. Após o fim da irrigação, os coeficientes de uniformidade aumentaram ao longo do tempo, em todas as profundidades estudadas; nas maiores profundidades, a distribuição foi mais uniforme, mesmo nos ensaios onde a uniformidade de água aplicada na superfície foi muito baixa. Em curtos intervalos de tempo, os coeficientes de uniformidade de umidade alcançaram altos valores. PERRENS (1984) relata que a intensidade de redistribuição da água no solo está inteiramente relacionada à textura. Avaliados e a uniformidade de umidade a $0,50 \mathrm{~m}$ de profundidade, durante o processo de redistribuição da água no solo, para um período de dois dias, a 
uniformidade aumentou de $60 \%$ para $67 \%$ em um solo de textura arenosa e de $60 \%$ para $73 \%$ em um solo tipo areno-siltoso.

\section{MATERIAIS E METODOS}

O trabalho foi realizado em um pomar de bananeira (BRS Princesa), localizado no campo experimental da Embrapa Mandioca e Fruticultura, no município de Cruz das Almas, situada no Recôncavo da Bahia, a $12^{\circ} 40^{\prime}$ '19' de latitude Sul, 39 $06^{\prime}$ ' 23' de longitude Oeste e altitude de $225 \mathrm{~m}$. O clima da região é classificado com úmido e Subúmido, com umidade relativa e temperatura média anuais de $80 \%$ e $24^{\circ} \mathrm{C}$, respectivamente, e pluviosidade média anual de $1.143 \mathrm{~mm}$ (D’ANGIOLELLA et al.,1998). O solo da área é classificado como Latosolo Amarelo álico Coeso de textura média (SOUZA \& SOUZA, 2001). Os estudos seguiram um delineamento de blocos casualizados em esquemas de parcelas subdivididas, com suas variáveis de cobertura (T5: com cobertura; T6: sem cobertura) na subparcela, profundidade $(0,20 ; 0,40 ; 0,60 \mathrm{~m})$ na subparcela e dois tempos ( 1 e 24 horas após a fertirrigação) na subparcela. Sendo o microaspersor utilizado com vazão de $53 \mathrm{Lh}^{-1}$ para quatro plantas, com espaçamento entre fileiras de $2,0 \mathrm{~m}$ x 2,5 m. Para as leituras de CEa e Umidade, foi feita a instalação de sondas de TDR nas profundidades $(0,20 ; 0,40 ; 0,60)$, em oito posições entre quatro touceiras de bananeira às distâncias radiais de 0,50 e $1,00 \mathrm{~m}$ da touceira, entre esta e o microaspersor. Coletores de água de aproximadamente $300 \mathrm{ml}$ foram dispostos em uma malha de $0,50 \mathrm{~m} \times 0,50 \mathrm{~m}$ na área ocupada pelas quatro plantas em cada tratamento antes da fertirrigação. A coleta de dados foi feita antes e após (1h e 24 h) uma fertirrigação ocorrida durante o ciclo da bananeira cultivar Princesa. Antes da fertirrigação foram distribuídos os coletores e feitas as leituras das sondas de TDR; após a fertirrigação, foram coletadas as laminas aplicadas em cada coletor bem como os volumes de água de irrigação foram acondicionados em frascos para posterior leitura de condutividade elétrica, o que foi feito com uso de um condutivimetro de bancada. Além disso foi determinado o $\mathrm{K}+$ e $\mathrm{NO}^{3}$ - com uso de um card Horiba. Os dados de lamina de água, concentração de potássio e nitrato da água de irrigação foram submetidos a seguinte equação (Bernardo et al., 2006):

$C U D=100 *[\mathrm{Iq} / \mathrm{Lm}]$

em que:

CUD = Coeficiente de uniformidade de distribuição do setor avaliado (\%); Iq = Média de $25 \%$ das observações com menores valores; $\mathrm{Lm}=$ Média dos valores encontrados.

Os valores de umidade e de condutividade elétrica lidos com uso da TDR $1 \mathrm{~h}$ e $24 \mathrm{~h}$ após nas três profundidades a fertirrigação foram submetidos a analise de variância e testes de média.

\section{RESULTADOS E DISCUSSÃO}

Os coeficientes de uniformidade de distribuição indicaram uma uniformidade baixa, classificada como inaceitável conforme Keller e Karmelli (1974) da lamina de água aplicada, que se deve ao efeito da distribuição localizada no entorno do microaspersor, pela desuniformidade de distribuição das gotas de água pelo emissor. Essa distribuição irregular não afeta a concentração da água de irrigação, resultando em um CUD maior, considerado bom (KELLER E KARMELLI, 1974) para o íon nitrato e normal para o potássio, o que mostra uma maior uniformidade de distribuição do nitrato em relação ao potássio na superfície do solo para as mesmas condições. Em valores absolutos, a uniformidade de distribuição de água e nutrientes não apresentou diferenças entre a quantidade de potássio ou nitrato na superfície do solo, na área de ocupação da bananeira com e sem cobertura morta (Tabela 1).

Tabela 1. Valores do coeficiente de uniformidade de distribuição (CUD), para lamina de água aplicada na superfície do solo e concentração de $\mathrm{K}+, \mathrm{NO}^{3}$ - na água de irrigação.

\begin{tabular}{cccc}
\hline Tratamento & NO3 & K & Lamina \\
\hline Com cobertura & 88,27484 & 78,018095 & 48,48 \\
Sem cobertura & 88,45256 & 74,85797 & 50,60 \\
\hline
\end{tabular}

A análise de variância para a umidade do solo uma hora após a fertirrigação identificou efeito do tratamento (cobertura morta) e da profundidade do solo, mas 24 horas após a fertirrigação, só foi 
detectado efeito da profundidade do solo na umidade do mesmo. Isso indica que a cobertura morta tem maior efeito na umidade do solo nas primeiras horas após a irrigação com redução do efeito com o tempo. No período de maior efeito a umidade sob a cobertura é superior a condição descoberta (Tabela 2). No caso da condutividade elétrica aparente do solo, não houve efeito da cobertura, apenas da profundidade do solo no período avaliado (tabela 1).

Tabela 2. Comparação de médias de umidade e de condutividade elétrica do solo nu e sob cobertura morta.

\begin{tabular}{cccc}
\hline $\begin{array}{c}\text { Tempo após a } \\
\text { fertirrigação }\end{array}$ & Tratamento & $\begin{array}{c}\text { Umidade } \\
\left(\mathrm{cm}^{3} \cdot \mathrm{cm}^{-3}\right)\end{array}$ & $\begin{array}{c}\text { Cond. Elétrica } \\
\left.(\mathrm{dSm})^{-1}\right)\end{array}$ \\
\hline $1 \mathrm{~h}$ & Com cobertura & $0,4079 \mathrm{~b}$ & 0,3933 \\
$1 \mathrm{~h}$ & Sem cobertura & $0,3050 \mathrm{a}$ & 0,3395 \\
$24 \mathrm{~h}$ & Com cobertura & 0,4162 & 0,3875 \\
$24 \mathrm{~h}$ & Sem cobertura & 0,3625 & 0,3354 \\
\hline
\end{tabular}

A distribuição de umidade no perfil 0-0,60 m indicou maior umidade no solo sob cobertura conforme verificado na tabela 1 , sendo que a exceção da profundidade de $0,20 \mathrm{~m} 1 \mathrm{~h}$ após a fertirrigação, todas as demais profundidades apresentaram umidades mais elevadas em relação ao solo sem cobertura. Esse resultado era esperado pelo efeito da cobertura em reduzir a evaporação de água do solo. No tempo de $24 \mathrm{~h}$ da fertirrigação a camada superficial do solo coberto foi a de maior umidade, o que pode ser devido a matéria orgânica mais abundante nessa camada em relação as demais e em relação ao solo nu.

Tabela 3. Comparação de médias de umidade e de condutividade elétrica do solo sob diferentes profundidades no solo nu e sob cobertura morta.

\begin{tabular}{ccrccc}
\hline $\begin{array}{c}\text { Tempo decorrido } \\
\text { da irrigação }\end{array}$ & Prof. do solo & \multicolumn{2}{c}{ Com cobertura } & \multicolumn{2}{c}{ Sem cobertura } \\
& $(\mathrm{m})$ & Umidade & CE & Umidade & CE \\
\hline \multirow{3}{*}{1 hora } & 0,20 & 0,4087 & $0,4600 \mathrm{a}$ & $0,4012 \mathrm{a}$ & $0,4612 \mathrm{a}$ \\
& 0,40 & 0,4162 & $0,4112 \mathrm{ab}$ & $0,3400 \mathrm{~b}$ & $0,3375 \mathrm{~b}$ \\
& 0,60 & 0,3987 & $0,3087 \mathrm{~b}$ & $0,3100 \mathrm{~b}$ & $0,2200 \mathrm{c}$ \\
\hline \multirow{3}{*}{24 horas } & 0,20 & 0,4437 & 0,4150 & $0,4075 \mathrm{a}$ & $0,4412 \mathrm{a}$ \\
& 0,40 & 0,4087 & 0,3925 & $0,3525 \mathrm{~b}$ & $0,3387 \mathrm{a}$ \\
& 0,60 & 0,3962 & 0,3550 & $0,3275 \mathrm{~b}$ & $0,2262 \mathrm{~b}$ \\
\hline
\end{tabular}

A condutividade elétrica de modo geral decresceu em profundidade, o que se justifica pelas fertirrigações que são aplicadas para suprir a camada da zona radicular. $O$ efeito da fertirrigação nessas profundidades é mais predominante no solo coberto onde se observa maior variação na CE de $1 \mathrm{~h}$ para 24 h após a fertirrigação, com redução da CE na camada de 0,20-0,40 m e elevação a 0,60 m, o que reflete maior descida dos íons comparada ao solo descoberto.

\section{CONCLUSÃO}

A uniformidade de distribuição para a lâmina na superfície obteve valores inaceitáveis pela literatura (Inferior a 50\%), os demais nutrientes obtiveram valores superiores ou próximos a $80 \%$ caracterizando uma boa distribuição. $\mathrm{O}$ solo com cobertura morta apresentou maior umidade, bem como maior variação da condutividade elétrica no perfil no período de 24 horas após a fertirrigação.

\section{REFERENCIAS}

ABNT- ASSOCIAÇÃO BRASILEIRA DE NORMAS TÉCNICAS. Sistema de irrigação localizada: Avaliação do desempenho. Rio de Janeiro, 11p, 1987. Projeto 12: 02-08-20.

ABNT-ASSOCIAÇÃO BRASILEIRA DE NORMAS TÉCNICAS. Sistema de irrigação por aspersão pivôcentral, caracterização de desempenho do método de ensaio; projeto de normas. Rio de Janeiro, 22p, 1985.(12:02.08.005) 
F. P. Santos et al.

AZEVEDO H.M. Irrigação localizada. Informe Agropecuário, Belo Horizonte, v.12, n.139, p.40-53, 1986.

BERNARDO, S. Manual de Irrigação, 8 ed. Viçosa: Imprensa universitária. 2008.393-396p.

ASAE - AMERICAN SOCIETY OF AGRICULTURAL ENGINEERS. Field evaluation of microirrigation systems. St. Joseph, p.792-797.1996.

BERNARDO,S.; SOARES, A. A.; MANTOVANNI,E.C Manual de Irrigação. 7. Ed. Viçosa: Universidade Federal de Viçosa,2005. 611p.

CRIDDLE, W.D.; DAVIS, S.; PAIR, C.H.; SHOCKLEY, D.G.Methods for evaluating irrigation systems. Washington DC: Soil Conservation Service - USDA, 1956. 24p. Agricultural Handbook, 82.

FRIZZONE, J.A. Irrigação por aspersão. Piracicaba: ESALQ-Departamento de Engenharia

Rural, 1992. 53p. Série Didática, 3.

JENSEN, M.E.; SWARNER, L.R.; PHELAN, J.T. Improving irrigation efficients. In. HAGAN, R.M. Irrigation of agriculture lands. St. Joseph: ASAE, 1967. Cap.61.p.1120-42(Agronomy,11).

LÓPEZ, J. R., ABREU, J. M. H., REGALADO, A. P., HERNÁNDEZ, J. F. G. Riego Localizado. Madrid,España: Mundi - Prensa, 1992. 405 p.

PAIVA, J.B.D. de. Uniformidade de aplicação de água, abaixo da superfície do solo, utilizando-se irrigação por aspersão. São Carlos: USP, 1980. 125p. DissertaçãoMestrado.

PERRENS, S.J. Numerical analysis of soil water uniformityunder sprinkler irrigation. Journal Agricultural

Engineering Research, Cambridge, v-30: p23-7, 1984.

REZENDE, R.; FRIZZONE; J.A.; BOTREL, T.B. Desempenho de um sistema de irrigação pivôcentral quanto à

uniformidade de distribuição de água, abaixo e acima da superfície do solo. Engenharia na Agricultura, Viçosa, v.2, n.19, p.01-07, 1992.

SANTOS, J.R.M. dos. Irrigar é preciso. Agroanalysis, Rio de janeiro, v.18, n.3. p.29-34, 1998.

SILVA, C. A.; SILVA, C. J. Avaliação de uniformidade em sistemas de irrigação localizada. Revista Cientifica Eletrônica de Agronomia, Garça, n.8, dez. 2005.

SOUZA, R. O. R. M. Desenvolvimento e avaliação de um sistema de irrigação automatizado para áreas experimentais. Piracicaba: ESALQ/USP, 2001. (Dissertação de Mestrado).

SOARES, A. A., RAMOS, M. M., LUCATO JÚNIOR, J. Uso racional de energia elétrica em sistemas de irrigação tipo pivô central no estado de Minas Gerais. In: CONGRESSO BRASILEIRO DE ENGENHARIA AGRÍCOLA, 22, 1993, Ilhéus-BA. Anais ... Ilhéus: 1993. p. 2688 - 2702.

KELLER,J.; KARMELI, D. Trickle irrigation design parameters. Transactions of the ASAE. St. Joseph, v.17, p.678-684,1974. 